\title{
Corrigendum: Innate control of actin nucleation determines two distinct migration behaviours in dendritic cells
}

Pablo Vargas, Paolo Maiuri, Marine Bretou, Pablo J. Sáez, Paolo Pierobon, Mathieu Maurin, Mélanie Chabaud, Danielle Lankar, Dorian Obino, Emmanuel Terriac, Matthew Raab, Hawa-Racine Thiam, Thomas Brocker, Susan M. Kitchen-Goosen, Arthur S. Alberts, Praveen Sunareni, Sheng Xia, Rong Li, Raphael Voituriez, Matthieu Piel and Ana-Maria Lennon-Duménil

Nat. Cell Biol. 18, 43-53 (2015); published online 7 December 2015; corrected online 23 December 2015

In the version of this Article originally published, references 42 and 43 were incorrect. They have been replaced with the following single reference: 42. Veltman, D. M. et al. PIP $_{3}$-dependent macropinocytosis is incompatible with chemotaxis. J. Cell Biol. 204, 497-505 (2014).

References 44-51 have been renumbered accordingly. These changes have been made in all online versions of the Article. 